\title{
LOCALLY EFFICIENT MONOTONE OPERATORS
}

\author{
ANDREI VERONA AND MARIA ELENA VERONA
}

(Communicated by William J. Davis)

\begin{abstract}
We study monotone operators on quasi open or convex subsets of a real Banach space $X$ (quasi open means that the contingent cone at each point equals $X$ ). Among others we characterize the maximality of such an operator in terms of its $\mathrm{w}^{*}$-upper semicontinuity properties and, in the case of a convex domain, also in terms of its behavior at the support points. We next give sufficient conditions for such an operator to be generically single valued, extending Kenderov's theorems. As an application we reobtain generic Gâteaux and Fréchet differentiability results for convex functions defined on not necessarily open convex sets.
\end{abstract}

\section{INTRODUCTION}

Let $X$ be a real Banach space. We shall denote by $X^{*}$ its topological dual, by $B(x, \varepsilon)$ the $\varepsilon$-ball in $X$ centered at $x$ and by $B^{*}$ the closed unit ball in $X^{*}$ (centered at the origin).

Let $A$ be a subset of $X$. For $x \in A$ let $K_{x}(A)$ be the set of all $y \in X$ such that there exist a sequence $\left(t_{n}\right)$ of positive real numbers convergent to 0 and a sequence $\left(y_{n}\right) \subset X$ convergent to $y$, with $x+t_{n} y_{n} \in A . K_{x}(A)$ is called the contingent cone to $A$ at $x$. It is well known that $K_{x}(A)$ is a closed cone and that $K_{x}(A)=K_{x}(\mathrm{cl}(A))$ (cl stands for the topological closure). If $C \subseteq X$ is convex and $x \in C$ then $K_{x}(C)=\operatorname{cl}\left(C_{x}\right)$, where $C_{x}=\{y \in X$ : there exists $t>0$ such that $x+t y \in C\}$ is the cone generated by $C$ from $x$. We shall denote by $C_{x}^{*}$ the normal cone to $C$ at $x$, i.e. $C_{x}^{*}=\left\{x^{*} \in X^{*}\right.$ : $x^{*}(x-y) \geq 0$ for any $\left.y \in C\right\}=\left\{x^{*} \in X^{*}: x^{*}(z) \leq 0\right.$ for any $\left.z \in C_{x}\right\}$.

Definition. A point $x \in A$ is called a quasi interior point of $A$ if $K_{x}(A)=X$; the set of all quasi interior points of $A$ is called the quasi interior of $A$ and is denoted qi $(A) . A$ is called quasi open if it is equal to its quasi interior.

Obviously any open set is quasi open. Other important examples of quasi open sets are those of the form $N(C)$, where $C$ is a convex set in $X$ and $N(C)$ denotes the set of all nonsupport points of $C$. Finally, any dense (respectively relatively open) subset of a quasi open set is quasi open.

Received by the editors February 12, 1988 and, in revised form, September 6, 1989.

1980 Mathematics Subject Classification (1985 Revision). Primary 47H05, 49A51, 58C20. 
Let us now recall some old definitions and introduce some new ones concerning monotone operators.

Definitions. Let $A$ be a subset of $X$.

(1) A subset $G \subseteq A \times X^{*}$ is called monotone if

$$
\left\langle x^{*}-y^{*}, x-y\right\rangle \geq 0 \text { for any }\left(x, x^{*}\right),\left(y, y^{*}\right) \in G .
$$

If in addition $G$ is maximal (under set inclusion) among all monotone sets contained in $A \times X^{*}$, then $G$ is called maximal monotone in $A \times X^{*}$.

(2) A multivalued map $T: A \rightarrow 2^{X^{*}}$, with $T(x) \neq \varnothing$ for all $x \in A$, is called a (maximal) monotone operator on $A$ if its graph $G(T)$ is a (maximal) monotone set in $A \times X^{*}$.

(3) Given a multivalued map $F: A \rightarrow 2^{X^{*}}$ and $r>0$ let $A_{r}=\{x \in A$ : $\left.F(x) \cap r B^{*} \neq \varnothing\right\}$ and define $F_{r}: A_{r} \rightarrow 2^{X^{*}}$ by $F_{r}(x)=F(x) \cap r B^{*} . F$ is called $r$-efficient at $a \in A$ if $F_{r}(x) \neq \varnothing$ for all $x$ in a relative neighborhood of $a$ in $A$. If $F$ is $r$-efficient at $a$ but we do not need to specify $r$, we shall say simply that $F$ is efficient at $a$. If for every $a \in A$ there exists $r_{a}>0$ such that $F$ is $r_{a}$-efficient at $a$, we call $F$ locally efficient.

(4) A multivalued map $F: A \rightarrow 2^{X^{*}}$ is called convex $w^{*}$-usco if $F(x)$ is a nonempty, compact and convex set in $X^{*}$ for each $x \in A$ and $F$ is norm-to$\mathrm{w}^{*}$ upper semicontinuous; if in addition its graph does not properly contain the graph of any other convex $\mathrm{w}^{*}$ - usco map on $A, F$ is called minimal.

Our first main result, stated below, provides a characterization of the maximal monotone operators among the locally efficient monotone operators on convex sets: a locally efficient monotone operator on a convex set $C$ is maximal monotone if and only if it is convex and $\mathrm{w}^{*}$-closed valued, $\mathrm{w}^{*}$-upper semicontinuous in a certain sense and, for every $x \in C, T(x)$ is invariant under translations by elements in the normal cone to $C$ at $x$. More precisely

Theorem A. Let $C \subseteq X$ be convex, $A \subseteq C$ be such that $K_{x}(A)=K_{x}(C)$ for each $x \in A$ and let $T: A \rightarrow 2^{X^{*}}$ be a locally efficient monotone operator. For every $x \in A$ choose $r_{x}>0$ such that $T$ is $r_{x}$-efficient at $x$. The following assertions are equivalent:

(1) $T$ is a maximal monotone operator on $A$;

(2) $T$ is convex and $w^{*}$-closed valued, $T_{r}$ is $w^{*}$-usco for every $r>0$ for which $A_{r} \neq \varnothing$ and $T(x)+C_{x}^{*} \subseteq T(x)$ for every $x \in A$;

(3) $T$ is convex and $w^{*}$-closed valued, $T_{r}$, is $w^{*}$ - usco at $x$ and $T(x)+C_{x}^{*} \subseteq$ $T(x)$ for every $x \in A$.

As a consequence we obtain the next corollary which generalizes the wellknown fact that a monotone operator on an open set is maximal monotone if and only if it is $\mathrm{w}^{*}$-usco.

Corollary I. Let $A \subseteq X$ be quasi open and $T: A \rightarrow 2^{X^{*}}$ be a locally efficient monotone operator. Then $T$ is maximal monotone if and only if it is convex $w^{*}$ - usco. 
The following result shows that monotone operators on certain domains have unique maximal monotone extensions (over the same domain). As above, in the case of open domains, this was known (see for example [5], Theorem 7.13).

Corollary II. Let $C \subseteq X$ be convex, $A \subseteq C$ be such that $K_{x}(A)=K_{x}(C)$ for each $x \in A$ and let $T: A \rightarrow 2^{X^{*}}$ be a locally efficient monotone operator. Then there exists a unique maximal monotone operator $M: A \rightarrow 2^{X^{*}}$ such that $T(x) \subseteq M(x)$ for every $x \in A$. If $A$ is quasi open on $X$ then $M$ can be described as follows: let $\widehat{T}$ be the monotone operator on $A$ whose graph is the closure (in $A \times X^{*}$ ) of the graph of $T$; then $M(x)$ is the $w^{*}$-closed convex hull of $\widehat{T}(x)$.

The next result may also be useful.

Corollary III. Let $T: A \rightarrow 2^{X^{*}}$ be a locally efficient maximal monotone operator and $A_{0} \subseteq A$ be such that $K_{x}\left(A_{0}\right)=K_{x}(A)$ for every $x \in A_{0}$. Assume that $A$ is either convex or quasi open. Then the restriction of $T$ to $A_{0}$ is maximal monotone (on $A_{0}$ ).

Theorem B. Let $A$ be a quasi open, Baire subset of the Banach space $X$ and let $T: A \rightarrow 2^{X^{*}}$ be a maximal monotone operator. Then there exists a dense $G_{\delta}$ subset $D$ of $A$ such that

(1) $T \mid D$ is a minimal convex $w^{*}$ - usco map;

(2) for each $x \in D, T(x)$ lies in a sphere of $X^{*}$ and $T$ is norm-to-w ${ }^{*}$-usco at $x$.

As a consequence we obtain the next corollary.

Corollary. Let $A$ be a quasi open Baire subset of the Banach space $X$ and let $T: A \rightarrow 2^{X^{*}}$ be a maximal monotone operator. Assume that $X$ is Asplund (resp. in Stegall's class $\mathscr{S}$ ). Then there exists a dense $G_{\delta}$ subset $A_{0}$ of $A$ such that $T$ is single valued and norm-to-norm (resp. norm-to- $w^{*}$ ) upper semicontinuous at each point of $A_{0}$.

Note. For an open $A$, the second assertion in Theorem B is due to Kenderov ([2]). He also proved the assertions in the last corollary in the following context: $A$ open and $X$ Asplund (resp. $X$ has an equivalent norm whose dual norm is strictly convex)(see [3] and [2]).

\section{LOCALLY EFFICIENT MONOTONE OPERATORS}

In this section we shall extend to our context some standard results in the theory of monotone operators, prove some new ones and use them to prove Theorems A and B.

Lemma 2.1. Let $A$ be a quasi open subset of $X, T: A \rightarrow 2^{X^{*}}$ be a monotone operator and let $a \in A$. Then $T$ is locally bounded at $a$ if and only if it is efficient at $a$. 
Proof. If $T$ is locally bounded at $a$, it is obviously efficient at $a$. Assume it is $r$-efficient at $a \in A$. Then there exists $\varepsilon>0$ such that $T(x) \cap r B^{*} \neq \varnothing$ for every $x \in B(a, \varepsilon) \cap A$. Let $x \in B(a, \varepsilon) \cap A$ and $y \in X$; since $x \in \mathrm{qi}(A)$ there exist a sequence $\left(t_{n}\right)$ of positive real numbers convergent to 0 and a sequence $\left(y_{n}\right) \subset X$ convergent to $y$ such that $x+t_{n} y_{n} \in A \cap B(x, \varepsilon)$. It follows that for each $n$ there exists $y_{n}^{*} \in T\left(x+t_{n} y_{n}\right) \cap r B^{*}$. Since $T$ is monotone, for every $x^{*} \in T(x)$ we have $\left\langle y_{n}^{*}-x^{*}, t_{n} y_{n}\right\rangle \geq 0$ which implies that

$$
x^{*}\left(y_{n}\right) \leq y_{n}^{*}\left(y_{n}\right) \leq r\left\|y_{n}\right\|
$$

and thus $x^{*}(y) \leq r\|y\|$. This shows that $\left\|x^{*}\right\| \leq r$ and therefore the lemma is proved.

Remark. A monotone operator is always locally bounded on the interior of its domain (see [7]; another proof, in a somewhat more general context, can be found in [1]).

Since we shall use it several times, let us recall the following well-known lemma.

Lemma 2.2. Let $A \subseteq X$ and $T: A \rightarrow 2^{X^{*}}$ be a monotone operator.

(1) $T$ is maximal if and only if $\left(x, x^{*}\right) \in A \times X^{*}$ and $\left\langle x^{*}-y^{*}, x-y\right\rangle \geq 0$ for all $\left(y, y^{*}\right) \in G(T)$ imply $x^{*} \in T(x)$.

(2) If $T$ is maximal, then $T(x)$ is a convex $w^{*}$-closed subset of $X^{*}$ for all $x \in A$.

Lemma 2.3. Let $C \subseteq X$ be convex and $A \subseteq C$ be such that $K_{x}(A)=K_{x}(C)$ for every $x \in A$ (e.g. $C=X$ and $A$ is quasi open). Let $T: A \rightarrow 2^{X^{*}}$ be $a$ locally efficient, convex, $w^{*}$-closed valued monotone operator. For every $x \in A$ let $r_{x}>0$ be such that $T$ is $r_{x}$-efficient at $x$ and assume that $T_{r_{x}}$ is $w^{*}$-usco at $x$. Assume also that $T(x)+C_{x}^{*} \subseteq T(x)$ for every $x \in A$. Then $T$ is $a$ maximal monotone operator.

Proof. Assume $T$ is not a maximal monotone operator. Then there exists $\left(y, y_{0}^{*}\right) \in A \times X^{*}$ such that $\left\langle x^{*}-y_{0}^{*}, x-y\right\rangle \geq 0$ for all $\left(x, x^{*}\right) \in G(T)$, but $y_{0}^{*} \notin T(y)$. Since $T(y)$ is convex and $\mathrm{w}^{*}$-closed, by the separation theorem there exist $u \in X$ and $\alpha \in R$ such that

$$
\left\langle y^{*}, u\right\rangle<\alpha<\left\langle y_{0}^{*}, u\right\rangle \text {, for all } y^{*} \in T(y) \text {. }
$$

First let us prove that $u \in K_{y}(A)=K_{y}(C)=\operatorname{cl}\left(C_{y}\right)$. Assume not. Then there exists $z^{*} \in X^{*}$ such that

$$
\left\langle z^{*}, x\right\rangle \leq 0<\left\langle z^{*}, u\right\rangle \text {, for all } x \in C_{y} .
$$

The left inequality implies that $z^{*} \in C_{y}^{*}$ and therefore $n z^{*} \in C_{y}^{*}$ for any positive $n$. Choose $y^{*} \in T(y)$. Then $y^{*}+n z^{*} \in T(y)$ and therefore $\left\langle y^{*}+\right.$ $\left.n z^{*}, u\right\rangle\left\langle\alpha\right.$ for any positive $n$, which implies that $\left\langle z^{*}, u\right\rangle \leq 0$, contradicting the choice of $z^{*}$. Thus $u \in K_{y}(A)$. 
Next let $r=r_{y}$ and choose $\beta \in R$ such that $\alpha<\beta<\left\langle y_{0}^{*}, u\right\rangle$. Let $W=$ $\left\{z^{*} \in X^{*} ;\left\langle z^{*}, u\right\rangle<\alpha\right\}$; since $W$ is $\mathrm{w}^{*}$-open and contains $T(y)$ and $T_{r}$ is $\mathrm{w}^{*}$ - usco at $y$, there exists a neighborhood $U$ of $y$ in $X$ such that $T_{r}(z) \subseteq W$ for any $z \in U \cap A$. We can also assume that $T_{r}(z) \neq \varnothing$ for all $z \in U \cap A$ (T is $r$-efficient at $y)$. Since $u \in K_{y}(A)$, there exist $t>0$ and $v \in V=\{z \in$ $\left.X ;\left\langle y_{0}^{*}, z\right\rangle>\beta\right\}$ such that $\|u-v\|<(\beta-\alpha) / r$ and $y+t v \in A \cap U$. Then $T_{r}(y+t v) \subseteq W$. For any $u^{*} \in T_{r}(y+t v) \subseteq W^{\prime}$ we have

$$
0 \leq\left\langle y_{0}^{*}-u^{*}, y-y-t v\right\rangle=-t\left\langle y_{0}^{*}-u^{*}, v\right\rangle
$$

and thus $\left\langle y_{0}^{*}, v\right\rangle \leq\left\langle u^{*}, v\right\rangle$. This implies that

$$
\left\langle u^{*}, u\right\rangle=\left\langle u^{*}, v\right\rangle+\left\langle u^{*}, u-v\right\rangle \geq\left\langle y_{0}^{*}, v\right\rangle-\left\|u^{*}\right\|\|u-v\| \geq \beta-r(\beta-\alpha) / r=\alpha
$$

and thus $u^{*} \notin W$, contradicting one of our previous choices. The lemma is therefore proved.

Lemma 2.4. Let $T: A \rightarrow 2^{X^{*}}$ be a maximal monotone operator and $\left(x_{n}\right) \subset A$ be a sequence norm convergent to $x \in A$. Let also $\left(x_{n}^{*}\right)$ be a sequence in $X^{*}$ with $x_{n}^{*} \in r B^{*} \cap T\left(x_{n}\right)$ (for some $\left.r>0\right)$. Then any $w^{*}$-cluster point of $\left(x_{n}^{*}\right)$ belongs to $T(x) \cap r B^{*}$ (and there are such cluster points!).

Proof. Since $r B^{*}$ is $\mathrm{w}^{*}$-compact, it follows that $\left(x_{n}^{*}\right)$ has $\mathrm{w}^{*}$-cluster points and all are contained in $r B^{*}$. Let $x^{*}$ be such a point. We need to show that $x^{*} \in T(x)$. Let $y \in A$ and $y^{*} \in T(y)$. We have $(*)$

$$
\begin{aligned}
\left\langle x^{*}-y^{*}, y-x\right\rangle & =\left\langle x^{*}-x_{n}^{*}, y-x\right\rangle+\left\langle x_{n}^{*}-y^{*}, y-x\right\rangle \\
& =\left\langle x^{*}-x_{n}^{*}, y-x\right\rangle+\left\langle x_{n}^{*}-y^{*}, y-x_{n}\right\rangle+\left\langle x_{n}^{*}-y^{*}, x_{n}-x\right\rangle .
\end{aligned}
$$

Since $x^{*}$ is a ${ }^{*}$-cluster point of $\left(x_{n}^{*}\right)$, by passing to a subsequence if necessary, we can assume that

$$
\lim \left\langle x^{*}-x_{n}^{*}, y-x\right\rangle=0 .
$$

Since $T$ is monotone,

$$
\left\langle x_{n}^{*}-y^{*}, y-x_{n}\right\rangle \leq 0 \text {. }
$$

Finally, since $\left(x_{n}^{*}-y^{*}\right)$ is bounded and $\left(x_{n}\right)$ is norm convergent to $x$, it follows that

$$
\lim \left\langle x_{n}^{*}-y^{*}, x_{n}-x\right\rangle=0 .
$$

Returning to $(*)$, we get

$$
\left\langle x^{*}-y^{*}, y-x\right\rangle \leq 0
$$

and Lemma 2.3 implies that $x^{*} \in T(x)$.

Corollary 2.5. If $T: A \rightarrow 2^{X^{*}}$ is a maximal monotone operator and $r>0$ is such that $A_{r} \neq \varnothing$, then $T_{r}$ is $w^{*}$-usco. In particular $T$ is $w^{*}$-usco at each point where it is locally bounded.

Proof. Assume that $T_{r}$ is not $\mathrm{w}^{*}$ - usco at a point $x \in A$. Then there exist a $\mathrm{w}^{*}$-neighborhood $W$ of $T_{r}(x)$, a sequence $\left(x_{n}\right) \subset A$ norm convergent to $x$ 
and a sequence $\left(x_{n}^{*}\right), x_{n}^{*} \in T_{r}\left(x_{n}\right)$, such that $x_{n}^{*} \notin W$ for all $n$. By Lemma 2.4, there exists a $\mathrm{w}^{*}$-cluster point $x^{*} \in T_{r}(x)$ of $\left(x_{n}^{*}\right)$. It follows that there exist $n$ 's such that $x_{n}^{*} \in W$. This contradiction proves the lemma.

We are now ready to prove Theorem $\mathrm{A}$ and its corollaries.

Proof of Theorem A. Assume that (1) is true. Then, by Lemma 2.2(2), $T$ is convex and $\mathrm{w}^{*}$ - closed valued. By Corollary 2.5, $T_{r}$ is $\mathrm{w}^{*}$ - usco for every $r>0$ for which $A_{r} \neq \varnothing$. Let $x \in A$ and $x^{*} \in T(x)+C_{x}^{*}$; from Lemma 2.2 (1) (using the maximality of $T$ and the definition of $C_{x}^{*}$ ), we obtain that $x^{*} \in T(x)$. Thus (1) implies (2). Obviously (2) implies (3). Finally, Lemma 2.3 shows that (3) implies (1).

Proof of Corollary I. One implication follows from Lemma 2.1 and Corollary 2.5 while the other one follows from the fact that $X_{x}^{*}=\{0\}$ for each $x \in X$ and Theorem A (more exactly from $(3) \Rightarrow(1)$ ).

Proof of Corollary II. By standard arguments there exists a maximal monotone extension $M$ of $T$. To prove that $M$ is unique, assume that there exists another maximal monotone operator $S$ on $A$ which contains $T$. Clearly $M \cap S: A \rightarrow 2^{X^{*}},(M \cap S)(x)=M(x) \cap S(x)$, is a convex, ${ }^{*}$ - closed valued monotone operator on $A$. It also satisfies $(M \cap S)(x)+C_{x}^{*} \subseteq(M \cap S)(x)$ for every $x \in A$ (since we can apply Theorem A to $M$ and $S$ ). Now let $r>0$ be such that $(M \cap S)_{r}$ is defined. Since $(M \cap S)_{r}$ has closed graph and is contained in $M_{r}$ which is w $^{*}$ - usco (by Theorem A), we can apply Proposition 7.5 in [5] to deduce that $(M \cap S)_{r}$ is w $^{*}$ - usco. Finally Theorem A implies that $(M \cap S)$ is a maximal monotone operator on $A$ and therefore $M=M \cap S=S$. This proves the uniqueness of $M$. The description of $M$ given in the last assertion of the corollary can be proved as in [5], Theorem 7.13.

The proof of Corollary III is obvious and we omit it.

Theorem 2.6. Let $A$ be a subset of the Banach space $X$ and let $T: A \rightarrow 2^{X^{*}}$ be a locally efficient maximal monotone operator. Then

(1) the function $\psi: A \rightarrow R$, defined by $\psi(x)=\inf \left\{\left\|x^{*}\right\| ; x^{*} \in T(x)\right\}$, is locally bounded and lower semicontinuous on $A$ (here $\left\|x^{*}\right\|$ represents the dual norm);

(2) the multivalued map $T_{0}: A \rightarrow 2^{X^{*}}$ defined by

$$
T_{0}(x)=\left\{x^{*} \in T(x):\left\|x^{*}\right\|=\psi(x)\right\}=T(x) \cap\left\{x^{*} \in X^{*}:\left\|x^{*}\right\| \leq \psi(x)\right\}
$$

is a locally bounded monotone operator and $T_{0}(x)$ is a nonempty, convex, $w^{*}$. compact subset of $X^{*}$;

(3) $T_{0}$ is $w^{*}$-usco at each point where $\psi$ is continuous.

Proof. (1) Since $T$ is locally efficient, $\psi$ is locally bounded. To prove the lower semicontinuity of $\psi$, let $\left(x_{n}\right)$ be a sequence in $A$, norm convergent to $x$. Let $U$ be a neighborhood of $x$ in $A$ and $r>0$ be such that $T(y) \cap r B^{*} \neq \varnothing$ for all $y \in U$. Then there exists $n_{0}>0$ such that $x_{n} \in U$ if $n>n_{0}$. For each 
$n>n_{0}$ there exists $x_{n}^{*} \in T\left(x_{n}\right)$ such that $\psi\left(x_{n}\right) \geq\left\|x_{n}^{*}\right\|-1 / n$ and $\left\|x_{n}^{*}\right\| \leq r$. By Lemma 2.4 the sequence $\left(x_{n}^{*}\right)_{n>n_{0}}$ has a ${ }^{*}$ - cluster point $x^{*} \in T(x) \cap r B^{*}$. Since the norm is $\mathrm{w}^{*}$-lower semicontinuous,

$$
\lim \inf \psi\left(x_{n}\right) \geq \liminf \left(\left\|x_{n}^{*}\right\|-1 / n\right) \geq\left\|x^{*}\right\| \geq \psi(x),
$$

showing that $\psi$ is lower semicontinuous at $x$.

(2) Let $x \in A$. The definition of $\psi$ implies that for every $n$ there exists $x_{n}^{*} \in T(x)$ such that $\psi(x)+1 / n \geq\left\|x_{n}^{*}\right\|$. This implies that $x_{n}^{*} \in(\psi(x)+1) B^{*}$ and therefore the sequence $\left(x_{n}^{*}\right)$ has a $\mathrm{w}^{*}$-cluster point $x^{*} \in T(x)$. Since

$$
\psi(x) \geq \liminf \left\|x_{n}^{*}\right\| \geq\left\|x^{*}\right\| \geq \psi(x)
$$

$x^{*} \in T_{0}(x)$, showing that $T_{0}(x) \neq \varnothing$. Clearly $T_{0}(x)$ is convex and $\mathrm{w}^{*}$ compact. Finally, $T_{0}$ is locally bounded because $\psi$ is.

(3) Let $\psi$ be continuous at $x$ and assume that $T_{0}$ is not $\mathrm{w}^{*}$-upper semicontinuous at $x$. Then there exists a ${ }^{*}$-neighborhood $W$ of $T_{0}(x)$ such that for every $n$ there exist $x_{n} \in B(x, 1 / n) \cap A$ and $x_{n}^{*} \in T_{0}\left(x_{n}\right)$ but $x_{n}^{*} \notin W$. Since $T_{0}$ is locally bounded, we may assume that $\left\|x_{n}^{*}\right\| \leq r$ for $n$ large and some $r>0$. By Lemma 2.4 there exists a $\mathrm{w}^{*}$-cluster point $x^{*}$ of $\left(x_{n}^{*}\right)$ contained in $T_{0}(x)$. Since $\psi$ is continuous at $x$ we have

$$
\psi(x)=\liminf \psi\left(x_{n}\right)=\liminf \left\|x_{n}^{*}\right\| \geq\left\|x^{*}\right\|
$$

and therefore $x^{*} \in T_{0}(x)$. Since $W$ is a $\mathrm{w}^{*}$-neighborhood of $x^{*}$ too, there exists $n$ such that $x_{n}^{*} \in W$, contradicting the choice of $W$ and $x_{n}^{*}$. Thus $T_{0}$ is $\mathrm{w}^{*}$-upper semicontinuous at $x$.

Note. The above theorem and its proof have been influenced by [2].

Since a lower semicontinuous function on a Baire space is continuous on a dense $\mathrm{G}_{\delta}$ subset of its domain, we obtain

Corollary 2.7. If $A$ is a Baire subset of $X$ and $T: A \rightarrow 2^{X^{*}}$ is a locally efficient maximal monotone operator then there exists a dense $G_{\delta}$ subset $D$ of $A$ such that $T_{0}$ is $w^{*}$-usco at each point of $D$.

Proposition 2.8. Let $A$ be a Baire subspace of $X$ and $T: A \rightarrow 2^{X^{*}}$ be a maximal monotone operator. Then there exists a dense, relatively open subset $D$ of $A$ such that $T$ is efficient at each point of $D$.

Proof. For each positive integer $n$ let

$$
F_{n}=\left\{x \in A ; T(x) \cap n B^{*} \neq \varnothing\right\} .
$$

Clearly $A=\bigcup F_{n}$. Observe that each $F_{n}$ is closed in $A$. Indeed, let $\left(x_{k}\right)$ be a sequence in $F_{n}$ norm convergent to $x \in A$. For each $k$ choose $x_{k}^{*} \in$ $T\left(x_{k}\right) \cap n B^{*}$. By Lemma 2.4, the sequence $\left(x_{k}^{*}\right)$ has a ${ }^{*}$-cluster point $x^{*} \in$ $T(x) \cap n B^{*}$, showing that $x \in F_{n}$. 
Now denote by $G_{n}$ the relative interior of $F_{n}$ in $A$. Then, since $A$ is Baire, $D=\bigcup G_{n}$ is dense in $A$ and of course relatively open. Obviously $T$ is efficient at each point of $D$.

We are now ready to prove Theorem B and its Corollary.

Proof of Theorem B. From the above proposition, there exists a dense, relatively open subset $D_{1}$ of $A$ such that $T$ is efficient at each point of $D_{1}$. By Corollary 2.7 there exists a dense $\mathrm{G}_{\delta}$ subset $D$ of $D_{1}$ such that, with the notation introduced there, $T_{0}$ is $\mathrm{w}^{*}$ - usco at each point of $D$. Since $D$ is quasi open in $X$, Corollary I implies that $T_{0}$ is a maximal monotone operator on $D$. However, by construction, $T_{0}(x) \subseteq T(x)$ for each $x \in A$ and therefore $T_{0}(x)=T(x)$ for $x \in D$. Finally, using again Corollary I, one can argue as in the proof of Theorem 7.9 in [5] and obtain that $T \mid D$ is a minimal convex $\mathrm{w}^{*}$ - usco map.

Proof of the corollary. When $X$ is Asplund, this can be proved as in the case of maximal monotone operators with open effective domains (see for example [5]). When $X$ is in Stegall's class $\mathscr{S}$ (see [8]), this follows directly from the definition of the class $\mathscr{S}$.

\section{APPLICATIONS TO THE DIFFERENTIABILITY OF CONVEX FUNCTIONS}

Let $C$ be a convex subset of the Banach space $X$ such that $\operatorname{claff}(C)=X$, $A$ be a relatively open subset of $C$ and $f: C \rightarrow R$ be convex.

Assume that $f \mid A$ is locally Lipschitz. Let $a \in A$ and let $M$ be a Lipschitz constant for $f$ on a neighborhood $U$ of $a$ in $A$. For $x \in U$ construct the continuous, sublinear function $F(x):, \operatorname{cl}\left(C_{x}\right) \rightarrow R$ as in [9] (recall that $F(x$,$) is defined on C_{x}$ by $F(x, v)=\lim _{t \downarrow 0}(f(x+t v)-f(x)) / t$ and then extended by continuity to $\left.\mathrm{cl}\left(C_{x}\right)\right)$. Observe that it is Lipschitz with Lipschitz constant $M$. The epigraph of $F(x$,$) and G=\{(v, r) \in X \times R ;-M\|v\|>r\}$ are disjoint convex subsets of $X \times R$ and, since $G$ is open, there exists a closed hyperplane separating them (and passing through $(0,0)$ ) given by a functional $\Phi \in(X \times R)^{*}$. Then there exist $\phi \in X^{*}$ and $a \in R, a<0$, such that $\Phi(v, r)=\phi(v)+a r$. A direct computation shows that $b \phi \in \partial F(x, 0)=\partial f(x)$ and $\|b \phi\| \leq M$, where $b=-1 / a$. Thus $f$ is subdifferentiable at every $x \in A$ and the subdifferential map $\partial f: A \rightarrow 2^{X^{*}}$ is locally efficient. Using arguments similar to those used in the proof of Lemma 2.4 and Corollary 2.5, one can prove that $(\partial f)_{r}$ is $\mathrm{w}^{*}$ - usco for every $r>0$. The definition of the subdifferential map also shows that $\partial f(x)+C_{x}^{*} \subseteq \partial f(x)$ for each $x \in A$. From Theorem A we obtain that $\partial f: A \rightarrow 2^{X^{*}}$ is a maximal monotone operator.

Conversely, if $\partial f(x) \neq \varnothing$ for each $x \in A$ and $\partial f: A \rightarrow 2^{X^{*}}$ is locally efficient, it is easy to see that $f \mid A$ is locally Lipschitz.

Before stating our last result, let us recall that the convex function $f: C \rightarrow R$ is called Gateaux differentiable at $x \in C$ if, with the above notation, $F(x$,$) is$ the restriction of a continuous linear functional $f_{x}^{\prime}$ on $X ; f$ is called Fréchet 
differentiable at $x \in C$ if there exists $f_{x}^{\prime} \in X^{*}$ such that

$$
\lim \left(f(y)-f(x)-\left\langle f_{x}^{\prime}, y-x\right\rangle\right) /\|y-x\|=0,
$$

as $y$ tends to $x$ in $C \backslash x$.

Proposition 3.1. Let $C$ be a convex subset of the Banach space $X$ such that $\operatorname{claff}(C)=X, A$ be a relatively open subset of $C$ and $f: C \rightarrow R$ be convex.

(1) $f \mid A$ is locally Lipschitz if and only if $\partial f(x) \neq \varnothing$ for every $x \in A$ and $\partial f: A \rightarrow 2^{X^{*}}$ is locally efficient; if this is the case, then $\partial f$ is a maximal monotone operator on $A$;

(2) if $A$ is a Baire space and $f \mid A$ is locally Lipschitz then there exists a dense $G_{\delta}$ subset $D$ of $A$ such that $\partial f(x)$ lies in a face of a sphere of $X^{*}$ for every $x \in D \cap N(C)$.

(3) if $X$ is Asplund (resp. of class $\mathscr{S}$ ), $A$ is Baire and $f \mid A$ is locally Lipschitz then there exists a dense $G_{\delta}$ subset $A_{0}$ of $A$ such that $f$ is Fréchet (resp. Gâteaux) differentiable at each point of $A_{0}$.

Proof. We already proved (1). The second assertion follows from Theorem B and the fact that $N(C)$ is a quasi open subset of $X$. To prove (3), notice that $A$ is paracompact (being a metric space). Using a partition of the unity argument and the fact that $\partial f$ is locally efficient one can construct a continuous function $\varphi: A \rightarrow R$ such that $\partial_{\varphi} f: A \rightarrow 2^{X^{*}}$ defined by $\left(\partial_{\varphi} f\right)(x)=\partial f(x) \cap \varphi(x) B^{*}$ is nonempty valued. As in the proof of Theorem 2.6 one can check that $\partial_{\varphi} f$ is a convex $\mathrm{w}^{*}$ - usco map. Let $T: A \rightarrow 2^{X^{*}}$ be a minimal convex ${ }^{*}$ - usco map whose graph is contained in the graph of $\partial_{\varphi} f$. Assume now that $X$ is Asplund. Then (see for example [5, Lemma 7.14 and Theorem 5.7]) there exists a selection $\sigma: A \rightarrow X^{*}$ for $T$ which is norm-to-norm continuous at each point of a dense $\mathrm{G}_{\delta}$ subset $A_{0}$ of $A$. Clearly $\sigma$ is a selection for $\partial f$ too. It remains to notice that the "if" part of Proposition 2.8 in [5] is also true in our context, namely: the existence of a norm-to-norm continuous at $x$ selection for the subdifferential map of $f$ implies the Fréchet differentiability of $f$ at $x$. The case when $X$ is of class $\mathscr{S}$ can be treated similarly.

Note. When $C$ is open, the above results are well known. For a not necessarily open $C$, parts of this proposition were previously proved by different methods in $[9,6,4$ and 10$]$.

Note. After this paper was accepted for publication, we noticed the following direct consequence of Theorem A:

Theorem $\mathbf{A}^{\prime}$. Let $C, A$ and $T$ be as in Theorem $\mathrm{A}$ and let $S: A \rightarrow 2^{X^{*}}$ be any locally bounded, convex valued, $w^{*}$-usco map such that $S(x) \subseteq T(x)$ for all $x \in A$ (such an $S$ always exists if $T$ is maximal monotone). Then $T$ is maximal monotone if and only if $T(x)=S(x)+C_{x}^{*}$ for all $x \in A$. 
This last resült is closely related to some results due to J. Borwein, S. Fitzpatrick and P. Kenderov (Minimal convex uscos and monotone operators on small sets, preprint, 1989).

\section{AKNOWLEDGMENT}

We would like to thank Professor R. R. Phelps for the useful remarks he made after reading a first version of this paper.

\section{REFERENCES}

1. J. M. Borwein and S. P. Fitzpatrick, Local boundedness of monotone operators under minimal hypotheses, Bull. Austral. Math. Soc. (to appear).

2. P. S. Kenderov, The set-valued monotone mappings are almost everywhere single-valued, C. R. Acad. Bulgare Sci. 27 (1974), 1173-1175.

3. __ Monotone operators in Asplund spaces, C. R. Acad. Bulgare Sci. 30 (1977), 963-964.

4. D. Noll, Generic Fréchet differentiability of convex functions on small sets, preprint (1987).

5. R. R. Phelps, Convex functions, monotone operators and differentiability, Lecture Notes in Math., vol. 1364, Springer-Verlag, 1989.

6. J. Rainwater, Yet more on the differentiability of convex functions, Proc. Amer. Math. Soc. 103 (1988), 773-778.

7. R. T. Rockafellar, Local boundedness of nonlinear monotone operators, Mich. Math. J. 16 (1969), 397-407.

8. C. Stegall, A class of topological spaces and differentiation of functions on Banach spaces, Proc. Conf. on Vector Measures and Integral Representations of Operators, Vorlesungen aus dem Fachbereich Math., Heft 10 (W. Ruess, ed.), Essen, 1983.

9. M. E. Verona, More on the differentiability of convex functions, Proc. Amer. Math. Soc. 103 (1988), 137-140.

10. $\ldots$ On the differentiability of convex functions, Proceedings of the Centre of Mathematical Analysis, vol. 20, Canberra, 1988, 195-202.

Department of Mathematics and Computer Science, California State University, Los ANGeles, CALIFornia 90032

Department of Mathematics, California Institute of Technology, Pasadena, CaliFORNIA 91125

Current address (Maria Elena Verona): Department of Mathematics and Computer SCIenCe, University of California, Riverside, California 92521 